\title{
Perspectivas sobre la enseñanza de la redacción académica para estudiantes de artes escénicas
}

\author{
Perspectives on the teaching of academic writing for \\ performing arts students
}

iD Gabriela Luisa Javier Caballero $\square$

Universidad Nacional Mayor de San Marcos. Lima, Perú

\begin{tabular}{llll}
\hline Recibido: 14/09/2019 & Revisado: 05/11/2019 & Aceptado: 08/01/2020 & Publicado: 18/06/2020
\end{tabular}

\section{RESUMEN}

La inserción a la vida académica representa una problemática para los ingresantes a la educación superior. Esta situación se agrava en el caso de los estudiantes de carreras relacionadas con las artes, ya que preexiste la concepción de que estas disciplinas están orientadas al desarrollo solo de capacidades artísticas; sin embargo, la vida universitaria supone también el desarrollo de otras capacidades relacionadas con la vida académica. A partir de estas dificultades, planteamos en este texto algunas perspectivas sobre la enseñanza de la redacción e investigación en el ámbito superior. Proponemos que esta debe articular el contenido propio de los cursos de la especialidad del estudiante con la práctica del investigador mediante escritos que evidencien la textualización de la práctica artística, conocimiento que resulta valioso en la especialidad.

Palabras clave: Redacción, investigación, artes escénicas, cultura académica.

\begin{abstract}
Starting academic life represents a problem to new comers into higher education. This situation is aggravated in the case of students in art-related majors, as there is a pre-conception that these disciplines are oriented towards the development of artistic skills only. However, university life also implies the development of other skills related to academic life. Considering these difficulties, we present in this text some perspectives about the teaching of essay writing and research at university level. We propose that the content of the student's major field of studies courses should be articulated with the research practice by means of writings that evidence the textualization of the artistic practice, knowledge that is valuable in the specialization.
\end{abstract}

Keywords: Essay writing, research, performing arts, academic culture. 


\section{INTRODUCCIÓN}

Uno de los principales retos con los que se enfrentan los estudiantes de artes escénicas (actuación, danza, diseño escenográfico) es la redacción académica. Una de las posibles causas de esta situación es que desconocen que en la actualidad, el perfil del artista escénico no se centra solamente en el paradigma del virtuosismo artístico, sino que la formación académica a nivel superior ${ }^{1}$ demanda un investigador creador. Este no solo debe manejar las técnicas vocales y físicas propias de su formación, sino que debe conocer estrategias lógicoracionales (análisis, comparación, producción de inferencias); retóricas (descripción, argumentación, explicación, persuasión) y formales (dominio de normativas del campo académico) para la exploración en torno a un problema de investigación previamente determinado y acotado.

Sobre este aspecto, resulta útil el acercamiento a la investigación en artes que propone Frayling (1997). El autor identifica tres tipos de investigación en relación con las artes. Uno de ellos se denomina investigación para las artes, la cual genera insumos para el trabajo artístico, por ejemplo, las indagaciones "que buscan generar tecnologías para el trabajo artístico" (Contreras, 2017). El siguiente tipo de investigación en artes es aquella que se realiza a través de las artes, que se diferencia de la anterior en tanto produce nuevo conocimiento en base a la creación, como una nueva técnica actoral, por ejemplo. La tercera es la investigación dentro las artes, en la que se trabaja con preguntas establecidas, hipótesis y con herramientas de aná- lisis que pueden provenir de otros campos del conocimiento, como la semiótica, los estudios culturales, etc. En una investigación de este tipo, la práctica artística es el objeto de estudio.

Por su parte, Borgdoff en el texto "El debate sobre la investigación en artes" propone, también, tres categorías para distinguir los tipos de investigación: la primera es la investigación en las artes ${ }^{2}$, aquella en la que no existe separación entre sujeto y objeto, entre el investigador y la práctica artística. Esta es el componente principal no solo del proceso de investigación, sino de los resultados que se generan. La segunda categoría es la de la investigación para las artes, que es aplicada y en la que, de modo similar a la que propone Frayling, el arte es el objetivo, pues aporta instrumentos que se usarán en propuestas escénicas. La tercera modalidad de investigación es sobre las artes, en la que, desde una distancia teórica, se extraen conclusiones válidas sobre una práctica artística. Sobre este tipo de investigaciones, sostiene Borgdorff: "es común en las disciplinas académicas de humanidades que se han ido estableciendo, incluida la musicología, la historia del arte, los estudios teatrales, los estudios de los medios de información y los de literatura" (2005: 9). Esta última, quizá, es la que está más ligada a lo tradicionalmente conocido como "académico", al "investigador tradicional". Sin embargo, en el marco de la reglamentación de la nueva Ley Universitaria, los estudiantes de los espacios académicos que ofrecen bachillerato y licenciatura deben graduarse

\footnotetext{
(1) En Lima, existen diversos espacios académicos que brindan formación en Artes Escénicas a nivel superior: La Facultad de Artes Escénicas de la Universidad Católica del Perú, la Escuela Nacional Superior de Arte Dramático, la Facultad de Ciencias Humanas de la Universidad Científica del Sur y la Universidad Nacional de Música. Podríamos incluir a la Escuela Nacional del Folklore.

(2) También ha sido denominada "investigación desde la práctica", "investigación basada-en-la-práctica", "investigación guiada-por-lapráctica" y "práctica como investigación" (Borgdorff, 2005: 10).
} 
con trabajos de investigación que validen sus habilidades como investigadores.

En ese sentido, como docentes de redacción e investigación académica, ¿cómo afrontamos esta situación? ¿Estamos preparados para complementar la formación como investigadores de los artistas escénicos? En este texto, planteamos una posible alternativa frente a esta problemática, a partir de nuestra experiencia como parte del equipo de la Dirección de Investigación de la Escuela Nacional de Arte Dramático (ENSAD), institución que implementó durante el 2016 2017 un programa de investigación orientado a mejorar las capacidades de redacción en el ámbito académico de sus estudiantes.

\section{Sobre el modelo de investigación en artes escénicas}

La investigación en artes escénicas puede ser diversa, y no solo implica lo práctico, en tanto existe una mirada desde el cuerpo y la creación, sino también procesos teóricos orientados a concretar las búsquedas que orientan la creación escénica. Para el desarrollo de la investigación artística, resulta fundamental hacer comprensible el conocimiento sensible producido mediante la exploración artística, con la finalidad de que sea valorado por la comunidad académica.

De acuerdo con Pérez Royo, existen dos modelos previos, tradicionales, de investigación en artes escénicas. Uno de ellos es el modelo educativo, según el cual "la formación artística se comprendía como un proceso de transformación y crecimiento espiritual global y espiritual del alumno basada en un énfasis igual en el cultivo de las dimensiones físicas, emocionales y sociales de la personalidad" (2010). Este tipo de formación estaría orientado no a que el estudiante "aprenda" ciertas técnicas o teorías, sino a que su personalidad se forme de tal modo que pueda producir artísticamente. Es decir, estaba más orientado al desarrollo de una sensibilidad particular, casi esencialista, sobre el arte: formar actores "sensibles" que generen experiencias estéticas. El otro modelo es el profesional, que "consiste en producir actores y bailarines profesionales de gran capacidad" (2010). En este, se valora el resultado, ya que el egresado de los centros de formación artística que se ciñen a este modelo debe crear propuestas escénicas o ser parte una propuesta escénica con nivel suficiente para expuesta ante un público. Estos egresados, deberían, idealmente, ser virtuosos en su campo: bailarines o actores profesionales.

Pérez Royo afirma que no basta con articular estos dos modelos educativos, sino que es necesario implementar una "transformación cualitativa definida por una orientación hacia la investigación, un énfasis en la producción de conocimiento y en la integración de la teoría y la práctica" (2010, p. 126). En el marco de esta problemática, en nuestra ciudad, durante los años 2016 y 2017, la Escuela Nacional Superior de Arte Dramático, con la finalidad de brindar a su comunidad académica implementó un programa de investigación que buscó, en primera instancia, brindar a los docentes las herramientas de investigación que les permitan academizar su trabajo y, en segunda instancia, introducir, mediante talleres, a los estudiantes a la práctica investigativa. En este texto, expondremos algunas de las ideas principales que guiaron dicha experiencia.

Un primer momento del programa de investigación estuvo enfocado en el 
cuerpo docente, pues no todos contaban con las herramientas propias del ámbito académico, sino que su formación, y la que brindaban a los estudiantes, estaba fundamentada en la práctica; es decir, en la enseñanza de técnicas vocales, actorales y de diseño escenográfico para que los egresados, de acuerdo al segundo modelo profesional que refiere Pérez Royo, tengan capacidades suficientes para ser parte de puestas en escena profesionales. El estatus universitario de la ENSAD determinaba que la práctica artística debía ser posible sistematizarse en un documento escrito. Dicho de otro modo, tanto docentes como estudiantes deberían escribir acerca de su propia práctica artística. En ese sentido, los profesionales de la Escuela Nacional Superior de Arte Dramático debían ser capaces de convertir sus experiencias creativas en conocimiento posible de ser difundido en el ámbito académico.

Los estudiantes, tanto de actuación como de diseño escenográfico ${ }^{3}$, mostraban algunas dificultades que les impedían desarrollar con solvencia la comprensión y producción de textos, ya que su formación estaba principalmente orientada al desarrollo de sus capacidades de creación. Como consecuencia de esto, al llegar al último año, tenían dificultades para escribir su tesis de grado, es decir, cuando tenían que escribir sobre su propia práctica artística. Debíamos aspirar a un modelo que articule lo práctico con la escritura académica.

Para esto, fue necesario contar con especialistas que acompañen este nivel de tareas académicas, pues la comunidad de la ENSAD no estaba familiarizada completamente con estas prácticas. El cuerpo docente del programa estuvo conformado por profesores y asistentes de docencia, en ambos casos, con formación en humanidades y con experiencia no solo en el dictado de cursos de este tipo, sino en investigación académica, es decir, contaban con tesis de licenciatura, artículos académicos publicados, etc. Este grupo de profesores tuvo que adecuar sus conocimientos, pues debían ayudar a los estudiantes a que organizaran la información sobre su práctica escénica, sobre la que luego escribirían. Por otro lado, la alianza con los docentes de las especialidades fue clave, ya que ellos son los portadores del saber específico de las disciplinas artísticas. Así, ellos orientaban a los estudiantes en lo respectivo a su curso en específico, mientras que el docente del taller de investigación guiaba la escritura sobre la práctica artística.

\section{La inserción de los estudiantes en la cultura académica}

La dirección general de la $\mathrm{ENSAD}^{4}$, con el objetivo de ofrecer a sus estudiantes una formación de calidad, solicitó a la dirección de investigaciones a cargo del profesor Percy Encinas, el diseño e implementación de la segunda etapa de del Programa de Inserción Académica (PINAC) que afronte la situación dada con los objetivos que conlleven a la excelencia académica de sus egresados. De acuerdo con Encinas (2016), en el programa concebimos a la cultura académica como:

El conjunto de prácticas, valores, hábitos y modos de relacionarse en una comunidad

(3) La ENSAD ofrece las carreras de Actuación, Diseño Escenográfico y Pedagogía. El programa solo se aplicó con las dos primeras especialidades.

(4) Durante ese periodo el director de la escuela fue Jorge Sarmiento Llamosas. 
profesional específica, según el campo de estudios. Esta cultura puede variar en distinta medida según la institución, el país o la época, pero por lo general comparte las principales características entre los parámetros de su campo. Por ejemplo, puede haber más diferencias entre la cultura académica de los veterinarios y los estudiantes de Literatura dentro de una misma universidad, en un país en el mismo año que entre la comunidad de Literatura de un país sudamericano y uno europeo, incluso en dos décadas distintas".

En ese sentido, se buscó introducir a los estudiantes en las prácticas académicas propias del campo, a saber, la lectura y la escritura y los mecanismos para optimizar ambas dinámicas. Estas son clave para el desempeño óptimo al interior de la comunidad universitaria. Además, están configuradas por normas generales y también específicas de su propio campo. Entre las normas generales que todo universitario debe manejar están las ortográficas o de normativa general, que usualmente son enseñadas durante los primeros ciclos universitarios al interior de cursos como lenguaje o redacción. Sin embargo, el PINAC no estuvo pensado como un taller de redacción o un curso que nivele el manejo del lenguaje de los estudiantes, sino que se buscó instalar "una relación directa y estrecha entre las prácticas letradas (lectura y escritura) que desea desarrollar en los estudiantes y los contenidos propios de su especialidad" (Encinas, 2016). Para este fin, se trabajó en torno a la articulación de los contenidos de los cursos de carrera, de modo que los estudiantes redactaran sobre su propia práctica.

Emplear los contenidos de los cursos hizo posible la motivación de los estudiantes, ya que se hallaban frente a problemas reales de su campo de estudio. Así, por ejemplo, los estudiantes de actuación 1 debían redactar sobre la aplicación de determinada técnica actoral para la construcción de un personaje. Esto los obligaba a reflexionar sobre su propia práctica artística y a evidenciar en el discurso escrito su proceso de creación. El nexo entre la práctica académica y el contenido de los cursos de especialidad fue clave.

El PINAC tuvo dos modalidades: el taller TICA (Taller de Introducción a la Cultura Académica), dirigido a estudiantes de los primeros ciclos $\left(1^{\mathrm{er}}, 2^{\mathrm{do}}, 3^{\mathrm{er}}\right)$ y TACIA (Taller de Comunicación e Investigación Académica, dirigido a quienes estaban en un estadio intermedio de su formación ( $5^{\text {to }}$ y $7^{\mathrm{mo}}$ ). Posteriormente, frente a la necesidad de asesorar a los alumnos que estaban por egresar que ya estaban redactando sus tesis, se implementaron asesorías académicas, bajo la modalidad de acompañamiento de un docente especialista en redacción académica que participaba en las clases de investigación actoral, así, este profesor conocía de cerca las investigaciones y las observaciones de los profesores especialistas que asesoraban los trabajos de grado.

En el taller TICA, se buscó que los estudiantes valoren la actividad del investigador como razón fundamental de su formación y que identifique temas posibles de ser objetos de investigación. Para lograr esto, se incluyeron algunos temas sobre las características del texto académico, el registro, estrategias lectoras y estrategias de redacción para que puedan describir y explicar. Las tareas académicas que se encomendaban en este taller tenían relación con sus cursos de especialidad. En 
el taller TACIA se buscó que el estudiante formule un problema de investigación pertinente y posible de ser desarrollado a lo largo de su formación universitaria, ya que en la ENSAD es usual que los estudiantes, a partir de $7^{\text {mo }}$ ciclo definan su tema de trabajo para graduarse. Eso debía ser redactado bajo la forma de un proyecto de investigación pertinente académicamente. Las asesorías académicas fueron sesiones en el profesor del PINAC comentaba, principalmente, la organización del trabajo y orientaba la redacción.

Esta experiencia demostró que, para una inserción en la cultura académica exitosa de estudiantes que no están familiarizados con este tipo de prácticas y, más aun, en carreras relacionadas con lo artístico, que son concebidas como espacios de formación de artistas con habilidades propias de su disciplina y no necesariamente de investigación o de redacción académica, resulta pertinente la articulación de las prácticas letradas con los temas de los cursos de especialidad, de modo que el estudiante se sienta motivado y que pueda, desde el inicio de su vida universitaria, constatar que la textualización de aquello que él hace sobre el escenario es conocimiento valioso, necesario de ser difundido.

\section{REFERENCIAS BIBLIOGRÁFICAS}

Alzate, L. y Parada, D. (2009). La investigación en las artes escénicas del Departamento de Teatro de la Facultad de Artes, de la Universidad de Antioquía. Revista Colombiana de las Artes Escénicas, 3, 22 - 38 .

Borgdoff, H. (2010). El debate sobre la investigación en las Artes. Cairon: revista de ciencias de la danza, 25 - 46.
Encinas, P. (2016). "Programa de inserción académica PINAC 2016". Documento redactado para la justificación del programa. Versión sin publicar.

Huamán, M. (2012). La formación humanista: Fundamentos y desafíos. Lima: Universidad Científica del Sur.

Eco, H. (1992). Los límites de la interpretación. Barcelona: Editorial Lumen.

Pérez, V. (2010). El perfil del investigadorcreador en los estudios de posgrado en Artes Escénicas en el Reino Unido. Cairon: revista de ciencias de la danza, 125 -138. 